Letter to the Editor

doi:10.1017/\$1041610216001599

\section{Aging with autism spectrum disorder: an emerging public health problem}

From 1943, when Leo Kanner originally described autism, and to the first objective criteria for "infantile autism" in DSM-III and the inclusion of Asperger's disorder in DSM-IV, the subsequent classification scheme for autistic disorders has led to a substantial change with the 2013 issuance of the DSM- 5 by including subcategories into one umbrella diagnosis of autism spectrum disorder (ASD) (Baker, 2013). ASD is a lifelong neurodevelopmental disorder, characterized by social and communication impairments and restricted, stereotypical patterns of behavior (Baker, 2013). It is currently expected that most, or all of the actual cases of ASD, are identified in a timely way (i.e. in early childhood). However, there are many undiagnosed older adults who may have met the current diagnostic criteria for ASD as children, but never received such a diagnosis due to the fact it had yet to be established. In addition, some patients with relatively less impairing phenotypes may escape formal diagnosis in childhood, only to later be diagnosed in adulthood. Nevertheless, the first generation of diagnosed patients with ASD is now in old age. Many such ASD patients have needed family and institutional support for their lives subsequent to childhood diagnosis. Due to aging and death of their parents and other supportive figures leading to a loss of social structures, there is no better time than now for the medical community to act.

There are two "telescoping" issues: (1) aging ASD patients who are clinically stable may now be bereft of familiar supportive figures and structures and will need alternative placements and supports, and (2) the "natural history" of aging with ASD and these patients' specific risk of acquired neurocognitive disorders (NCDs; formerly dementia) (e.g. degenerative or vascular dementia), which has yet to be fully elucidated. Clinical interventions specific to this complex aging population need to be developed and validated. More research about aging with ASD with a focus on transition from middle adulthood to older age, management of social cognition impairments, management of systemic medical comorbidity, and quality of life and support system issues, is greatly needed. Clinicians who regularly work with patients experiencing physiological aging (e.g. geriatric medicine, geriatric psychiatry, consultation-liaison psychiatry) may not have a full understanding of the childhood aspects of ASD (which is often thought of as primarily a childhood disorder), whereas clinicians working with ASD patients (e.g. pediatrics, developmental pediatrics, child neurology, child and adolescent psychiatry) do not necessarily have clinical currency in problems of aging (unless they are following ASD patients throughout the life span) since they do not typically treat geriatric patients. Bringing awareness of this imminent wave of "graying ASD" is required for medical care delivery systems to provide expert care for those aging with ASD. To support this endeavor, more research on the natural history of ASD with aging and an appreciation of its relative stability versus deterioration with aging is critical.

Although overt symptoms of ASD become established in the early developmental period, core difficulties tend to persist through life, often in a less severe form with increasing age. Geriatric patients with ASD will not typically reach normal levels of social functioning (Geurts and Vissers, 2012; Baker, 2013). Nevertheless, little is known about its geriatric clinical presentation and comorbidity. Recognizing previously undiagnosed ASD in geriatric patients, with or without comorbid neuropsychiatric syndromes, is imperative. James et al. (2006) presented a case series of geriatric patients (aged 67-84 years) believed to have met the criteria for Asperger's disorder. They argued that recognizing and diagnosing ASD in those patients facilitated their treatment, especially because these patients originally were misdiagnosed with mood and anxiety disorders, deemed as "treatment resistant", and may have received unnecessary, lengthy clinical psychiatric care that was not directed toward their core illness of ASD. James et al. (2006) suggested that for older patients with a history of social skills problems evident from early childhood, clinicians should routinely take a lifelong history in understanding patients' interpersonal deficits, particularly in those geriatric patients with a longstanding, "detached" premorbid personality style, chronic social anxiety, difficulties in coping with change, and unusual behaviors of rigid routines, which may contribute to the profile in support of a new diagnosis of ASD in an older patient.

Research on the cognitive profile of ASD has been mainly restricted to childhood and early adulthood subjects even though physiological 
aging is known to have a strong impact on cognitive functioning (Geurts and Vissers, 2012). Since executive dysfunction is also commonly reported in ASD (Geurts and Vissers, 2012), neuropsychological findings in the geriatric ASD patients have to be interpreted with caution as dysexecutive phenomena are also present in NCD syndromes. Moreover, the effect of aging was found to differ between patients with and without high functioning ASD (Geurts and Vissers, 2012). How the interaction of the aging process and ASD affects resultant cerebral structure and functioning, and thereby cognition and behavior, is yet undetermined. A study focusing on cerebral structure in patients with ASD (aged 10-60 years) showed that age-related reduction in cortical volume seen in specific areas of healthy persons is less profound in those with ASD (Raznahan et al., 2010), which might potentially suggest a smaller age-related decrease in cognitive function in older ASD patients. Whether ASD patients develop compensatory cognitive mechanisms throughout life and/or experience progressive cognitive deterioration remains to be elucidated.

Advanced age is one of the risk factors for NCDs, but little is known about the prevalence of these acquired disorders in geriatric ASD patients. It is largely unknown whether aging patients with ASD are more (e.g. as in Down syndrome), less, or similarly predisposed to developing NCDs when compared to the general population of geriatric patients. Other risk factors typically associated with NCDs appear to also constitute systemic medical comorbidity of ASD (e.g. seizure disorders, depression, metabolic disorders, sensory dysfunction) (Bauman, 2010), which could predispose ASD patients to developing comorbid NCDs. Whether the established prevention strategies for NCDs may play a role in potential cognitive preservation in ASD remains to be ascertained.

Multidisciplinary assessment and coordination of clinical and social interventions for the patient with ASD are needed throughout the life span. Clinicians, families, caretakers, and friends should be involved in these processes, as appropriate. This may subserve mitigating the risk of isolation and social exclusion. Moreover, the Internet has helped ASD persons bypass their issues with social communication and, more broadly, the social cognition difficulties of this population, and has proven to be an effective platform to work remotely or form online communities (Biever, 2007). Since digital communication may be a more effective method to reach out to this "invisible" aging population, medical systems (particularly primary care clinicians) may wish to explore the use of "virtual" outreach consultations using telemedicine and similar technological applications rather than standard in-person face-to-face outpatient appointments.

In conclusion, little is known about ASD and its clinical presentation and comorbidity with NCDs in the geriatric population. Although dysexecutive syndromes are commonly reported in ASD, the exact risk of developing dementing illness per se complicating ASD in geriatric patients remains to be elucidated. Nevertheless, we argue that there is a great need, even for older adults, to receive an accurate diagnosis of ASD. This could benefit their treatment especially as they could otherwise inaccurately be diagnosed and treated as "treatment-resistant" depressive and/or anxiety disorders, largely because of misinterpreting some ASD symptoms (e.g. psychomotor agitation, anxious affect). Some warning signs that an older adult may have undiagnosed ASD include a chronic history of social anxiety, pervasive social skills problems, and esoteric ritualistic behaviors and routines (e.g. repetitive, focused, rigid and limited range of social interests and activities). These symptoms are not individually specific for ASD in isolation, but can suggest a diagnosis of ASD when present as a characteristic constellation of symptoms, thus supporting a diagnosis of ASD. Aging patients with ASD are in need of a high level of support, at least due to problems with social communication and interaction, may have a double "cognitive vulnerability" (due to baseline ASD plus secondary dementing illness), and may face a high risk of social isolation. Studies on the efficacy and safety of psychopharmacological interventions in geriatric patients with ASD are needed, particularly in regard to cholinesterase inhibitors (which have an increased risk of seizures), or antipsychotics (which have an increased risk of metabolic syndrome and cognitive impairment). In light of the rapidly growing geriatric population, coupled with the considerable lack of societal awareness of the realities of aging in ASD, now is the time for researchers to turn their attention to investigating the needs of geriatric ASD.

\section{Conflict of interest}

None.

\section{Acknowledgments}

None. 


\section{References}

Baker, J. P. (2013). Autism at 70 - redrawing the boundaries. New England fournal of Medicine, 369, 1089-1091.

Bauman, M. L. (2010). Medical comorbidities in autism: challenges to diagnosis and treatment. Neurotherapeutics, 7, 320-327.

Biever, C. (2007). Web removes social barriers for those with autism. New Scientist, 2610, 26-27.

Geurts, H. M. and Vissers, M. E. (2012). Elderly with autism: executive functions and memory. Fournal of Autism and Developmental Disorders, 42, 665-675.

James, I. A., Mukaetova-Ladinska, E., Reichelt, F. K., Briel, R. and Scully, A. (2006). Diagnosing Aspergers syndrome in the elderly: a series of case presentations. International fournal of Geriatric Psychiatry, 21, 951-960.
Raznahan, A. et al. (2010). Cortical anatomy in autism spectrum disorder: An in vivo MRI study on the effect of age. Cerebral Cortex, 20, 1332-1340.

\author{
Ana Hategan, ${ }^{1}$ James A. Bourgeois ${ }^{2}$ \\ AND JEREMY GOLDBERG ${ }^{1}$ \\ ${ }^{1}$ Department of Psychiatry \& Behavioural \\ Neurosciences, Michael G. DeGroote School of \\ Medicine, Faculty of Health Sciences, McMaster \\ University, Hamilton, Ontario, Canada \\ ${ }^{2}$ Department of Psychiatry/Langley Porter Psychiatric \\ Institute, Consultation-Liaison Service, University of \\ California San Francisco Medical Center, San \\ Francisco, California, USA
}

